\title{
Peptide-Based Materials for Cartilage Tissue Regeneration
}

\author{
Nurcan Hastar, Elif Arslan, Mustafa O. Guler, \\ and Ayse B.Tekinay
}

\begin{abstract}
Cartilaginous tissue requires structural and metabolic support after traumatic or chronic injuries because of its limited capacity for regeneration. However, current techniques for cartilage regeneration are either invasive or ineffective for long-term repair. Developing alternative approaches to regenerate cartilage tissue is needed. Therefore, versatile scaffolds formed by biomaterials are promising tools for cartilage regeneration. Bioactive scaffolds further enhance the utility in a broad range of applications including the treatment of major cartilage defects. This chapter provides an overview of cartilage tissue, tissue defects, and the methods used for regeneration, with emphasis on peptide scaffold materials that can be used to supplement or replace current medical treatment options.
\end{abstract}

\section{Keywords}

Cartilage $\bullet$ Cartilage regeneration - Scaffold types $\bullet$ Peptide scaffolds • Self-assembly

N. Hastar • E. Arslan

Institute of Materials Science and Nanotechnology,

National Nanotechnology Research Center (UNAM),

Bilkent University, Ankara 06800, Turkey

M.O. Guler

Institute for Molecular Engineering, University of

Chicago, Chicago, IL 60637, USA

Institute of Materials Science and Nanotechnology,

National Nanotechnology Research Center (UNAM),

Bilkent University, Ankara 06800, Turkey

\author{
A.B. Tekinay $(\bowtie)$ \\ Institute of Materials Science and Nanotechnology, \\ National Nanotechnology Research Center (UNAM), \\ Bilkent University, Ankara 06800, Turkey \\ Neuroscience Graduate Program, Bilkent University, \\ Ankara 06800, Turkey \\ e-mail: atekinay@bilkent.edu.tr
}




\subsection{Introduction}

Cartilage is a connective tissue that structurally supports the body and assists in movement by creating low-friction platforms between the articular spaces of bone. Cartilaginous tissue does not contain any vascular and nervous elements, which severely limits its capacity for regeneration following injury (Ateshian 2007). Microfracture and autograft transplantation techniques are the current medical standards for the repair of cartilage damage. However, these methods are not fully capable of restoring the structural and functional integrity of cartilage tissue, necessitating the development of alternative procedures. The extracellular matrix (ECM) is responsible for the lubrication and articulation functions of cartilage. Therefore, ECM-mimetic intelligent and tunable scaffolds can serve as a temporary replacement for cartilage tissue while supplying the environmental conditions necessary for its regeneration. Modified polymers and peptides can also be utilized for the recruitment of mesenchymal stem cells to the damaged area, thereby promoting the formation of neo-cartilage tissue. However, these scaffolds must be biocompatible and biodegradable in order to minimize immunogenic responses, stimulate cell proliferation and integrate effectively with the surrounding tissue. In addition, they should have a porous structure to facilitate cellular migration and communication, and exhibit mechanical properties resembling native cartilage tissue to support newly formed tissues and provide the necessary signals for cellular recruitment and differentiation.

This chapter presents the features of cartilage tissue, its diseases, and the methods developed for promoting its regeneration. A broad overview of the advantages and disadvantages associated with both recent and established treatment options are discussed to provide further focus on the use of peptide bearing polymers and self-assembled peptide gels for cartilage engineering.

\subsection{Cartilage Tissue}

\subsubsection{Structure of Cartilage Tissue}

Cartilage tissue has a limited capacity for regeneration due to its avascular, aneural and alymphatic nature, which prevents the influx of oxygen, nutrients and biochemical signals that are required for effective wound repair. In addition, cartilage regeneration occurs through the activity of native chondrocytes and mesenchymal stem cells (MSCs), which have sparse populations and cannot facilitate the complete repair of large defects. Consequently, cartilage has a tendency to accumulate partially repaired defects with age, which progressively impairs tissue function and may result in degenerative joint diseases such as osteoarthritis, severely lowering the quality-of-life in the elderly population in particular.

Cartilage tissue is composed of cartilagespecific cells (chondrocytes) that constitute 3-5\% of the adult articular cartilage by mass and are embedded in a highly dense ECM (Han et al. 2011). MSCs are also present in cartilage tissue, especially in the deep part of the perichondrium, where they encircle the cartilage and may differentiate into chondroblasts (Slomianka 2009). However, cartilage is mostly composed of ECM elements, and load-bearing, the main function of cartilage tissue, is provided mainly by the macromolecular components of dense ECM (Taipaleenmaki 2010). In addition to its mechanical integrity, cartilage tissue also provides joint lubrication and articulation, and the ECM microenvironment likewise plays crucial roles in these functions (Han et al. 2011). The cartilage ECM is composed mainly of a fibrillar collagen network that is supported by various proteoglycans (Han et al. 2011). Among the collagen fibrils, type II collagen is the primary structural component found in cartilage ECM. Other cartilage-associated collagens include types III, VI, IX, X, XI, XII and XIV, which all contribute to the mature extracellular microenvironment (Eyre 2002). Besides collagen fibrils, the cartilage ECM also contains proteoglycans such as aggrecan, chondroitin sul- 
fate glycosaminoglycan, keratin sulfate glycosaminoglycan, hyaluronan, and glycoprotein lubricin (PRG4) (Lin et al. 2005). These cartilage-specific proteins are responsible for establishing the morphology and function of the tissue, and their malfunction contributes to the secondary expansion of cartilage defects. Consequently, the induction of proteoglycan synthesis is a promising approach for promoting the recovery of cartilage injuries.

\subsubsection{Cartilage Diseases}

Osteoarthritis is an inflammatory disease that is associated with the deterioration and erosion of articular cartilage, and occurs in response to a combination of genetic, metabolic and biochemical factors (Felson et al. 1997). The osteoarthritis $(\mathrm{OA})$ is a pathological condition involving the interactions of cartilage, bone and synovium. Aging (and the associated mechanical wear in joints) is the main cause for osteoarthritis and other degenerative cartilage disorders, although joint problems can also manifest following traumatic accidents and in obese persons (Grotle et al. 2008). One major problem in the treatment of OA and similar diseases is the progressive loss of tissue integrity resulting from day-to-day activity and the bodily weight, as cartilage exhibits a minimal capacity of self-renewal that can easily be overwhelmed by such factors. Consequently, the pathophysiological formation of $\mathrm{OA}$ is predominantly due to the destabilization of the anabolic and catabolic dynamics of cartilage, which results in the progressive destruction of the tissue (Moreland 2003) This process is regulated by cytokines, growth hormones and enzymes (aggrecanases, matrix metalloproteinases, etc.) synthesized by chondrocytes and synovial cells. During OA formation, collagen degradation increases, proteoglycan content decreases, types of macromolecules change, and water content increases (Fig. 7.1, Heinegård and Saxne 2011; Belcher et al. 1997; Brandt et al. 2000; Moreland 2003). In addition, the amount and molecular size of hyaluronic acid in the synovial fluid are also reduced (Belcher et al.
1997). Such progressive degeneration is a very invasive process, and early intervention is key for its management: radiological osteoarthritic findings may develop in less than 10 years when the patients are not treated during the early period of injury (Prakash and Learmonth 2002). Even small $(\sim 10 \mathrm{~mm})$ defects can result in the reduction of $50 \%$ or more of cartilage tissue in the joint, while larger $(>10 \mathrm{~mm})$ defects are known to provide an additional burden of $64 \%$ to the surrounding cartilage, as observed in a 14-year follow up study (Kock et al. 2008). Due to their severe and long-lasting effects, the treatment of cartilage diseases has been a subject of intensive study, although effective methods for promoting long-term tissue repair are yet to be discovered. Therefore, there is an urgent medical need for alternative treatment options and new multifunctional biomaterials that are able to respond to cartilage damage in a short period of time.

\subsubsection{Clinical Treatment Strategies for Cartilage Regeneration}

The microfracture technique is one of the most common treatment methods against cartilage damage. This method is based on the introduction of mesenchymal stem cells from lower bone into cartilage defects following the induction of local bleeding with the help of small needles. However, in the absence of a supportive artificial matrix, stem cells recruited in this manner generate fibrous tissue formations that are structurally and biomechanically dissimilar to the natural architecture of cartilage. In addition, successful treatment requires an accurate assessment of the extent of cartilage damage. In the clinic, arthroscopic debridement and lavage are performed for patients with less than $2 \mathrm{~cm}^{2}$ of cartilage damage, as this method involves the cleaning of the site of injury and minimizes local pain resulting from frictional forces acting on damaged cartilage. This treatment method is generally more suitable for elderly patients, who are less mobile and do not run the risk of causing further damage to the site of injury through active movement (Gaissmaier et al. 2008). In contrast, 

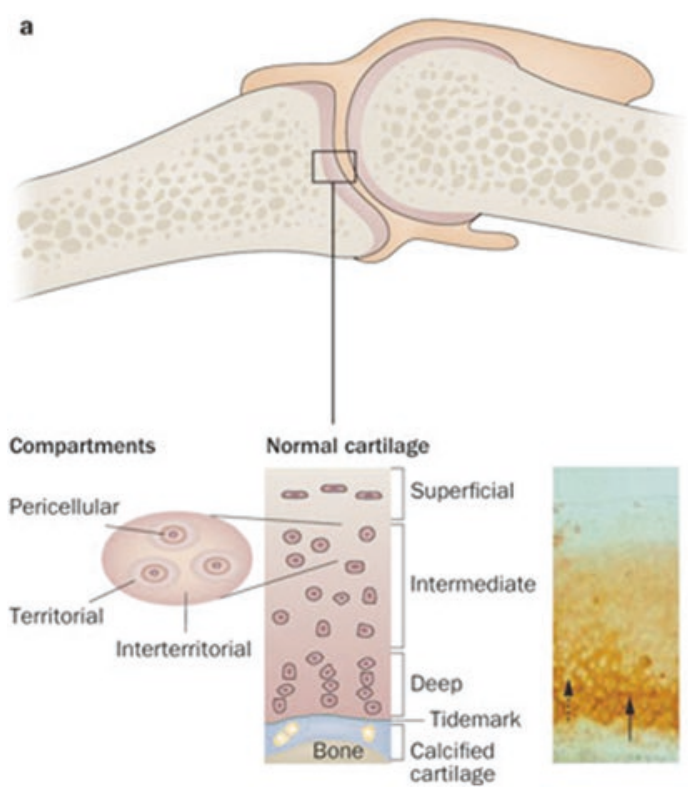

Fig. 7.1 (a) Representation of healthy joint of articular cartilage. Pericellular, territorial and interterritorial matrices form articular cartilage and each of them place at particular distance from the chondrocytes. Inset image indicates the immunohistochemistry of cartilage oligomeric matrix protein (COMP). While the territorial and pericellular matrices (dashed arrow) did not stained, the interterritorial matrix stains for COMP (solid arrow). (b) Representation of osteoarthritic joint. Although disease is

the microfracture method is preferred for defect sizes in the range of $2-3 \mathrm{~cm}^{2}$. This method is especially suitable for stimulating the migration of MSCs from bone marrow to cartilage, allowing new cartilage formation in full-thickness cartilage injuries (Kreuz et al. 2006). However, effective cartilage repair in this method is contingent upon the minimization of movement and mechanical loading at the affected site. In addition, mid- and long-term follow-up studies have shown that hyaline cartilage formation is limited and the newly formed tissue is dominated by fibrous cartilage following microfracture surgery: Although a hyaline-like cartilaginous matrix is initially formed at the site of injury, this material eventually acquires a fibrous character and deteriorates due to its inadequate mechanical and viscoelastic properties. Consequently, the joint gradually loses its functionality following surgery and must be replaced by prosthetics ( $\mathrm{Li}$

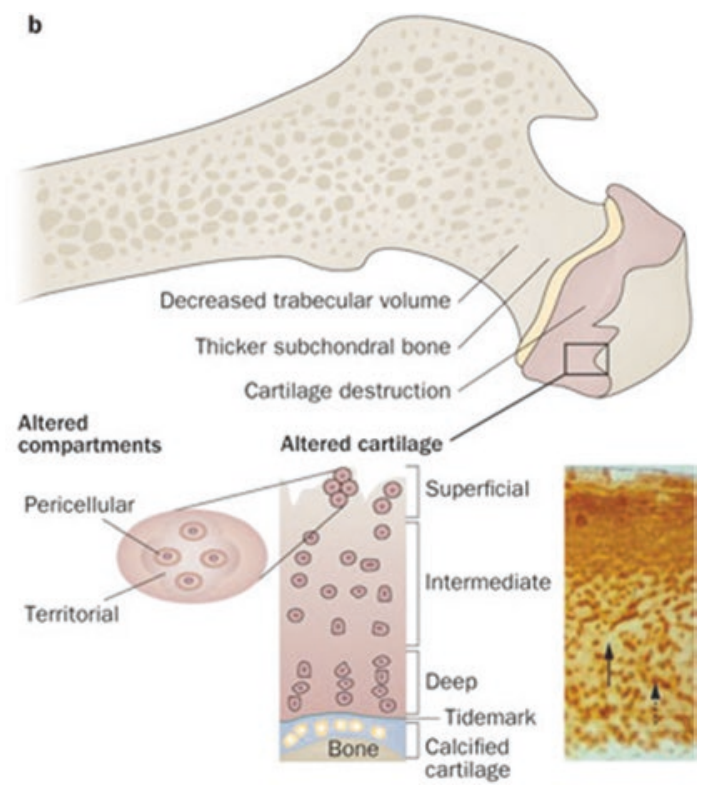

at early stages, degeneration of cartilage partially, alteration in the underlying bone and cloning and reproduction of cells were observed in joints. No immunohistochemistry staining for COMP at interritorial matrix (solid arrow) and storage of newly synthesized COMP at pericellular matrix of the cartilage (dashed arrow) demonstrate the cloning of cells is not distinct yet (Reprinted from Heinegård and Saxne 2011)

et al. 2009). In this context, cell and tissue implantation studies are generally applied as a secondary treatment method in patients with cartilage damage greater than $2 \mathrm{~cm}^{2}$. This treatment method is performed by transplanting chondral and osteochondral autografts in patients who do not respond well to lavage and microfracture treatments. In larger injuries, damaged cartilage tissue from non-load bearing joint regions may also be implanted. An important advantage of this method is the use of healthy, functional cartilage tissue, which can be obtained through arthroscopy. However, the implantation further damages the healthy tissue that surrounds the defect site, preventing the uniform integration of the autograft (Gillogly et al. 1998). Considering the disadvantages of these clinically applied treatment modalities, alternative treatment methods are needed for the effective recovery of cartilage injuries. Tissue engineering is a new 
and promising approach to enhance cartilage tissue regeneration, and a variety of biomaterials and cell culture methods have been developed to eliminate the deficiencies associated with conventional methods of cartilage repair.

\subsection{Biomaterials for Cartilage Regeneration}

Since cartilage has a limited capacity for regeneration, cells require additional biochemical and mechanical signals to effectively facilitate defect closure. Biomaterial scaffolds are next-generation tools for tissue engineering studies, and provide a combination of these signals in a 3D microenvironment to promote cellular attachment and proliferation. In addition, the highly porous structure of biomaterial scaffolds allows them to mediate the cell-cell interactions and signaling pathways that assists in cartilage repair (Ge et al. 2012). Biocompatibility and biodegradability are indispensable characteristics of all biomaterials designed for tissue applications, while flexibility, elasticity and customizable mechanical strength are required to emulate the natural environment of cartilage in particular (Pearle et al. 2005).

\subsubsection{Natural and Synthetic Scaffolds}

A broad range of both natural and synthetic biomaterials have been used to support cell adhesion, viability, proliferation, and differentiation in cartilage tissue engineering studies. Natural scaffolds can be extracted from live organisms and include materials such as collagens, GAGs and decellularized cartilage of animal origin. Foremost among these materials is hyaluronic acid, which is a carbohydrate-based, non-sulfated glycosaminoglycan that is found abundantly in cartilage tissue and promotes chondrocyte proliferation as well as chondrogenesis in MSCs. Many applications of this material exist in the literature: For example, a hyaluronic acid derivative biopolymer (Hyaff®-11) was seeded with autologous chondrocytes and shown to enhance the quality of newly formed cartilage tissue following its direct injection into full-thickness cartilage defects (Grigolo et al. 2001). Collagens (mostly type II) are also common in the natural cartilage ECM, and see frequent use in tissue engineering applications due to their lack of toxicity, ability to integrate rapidly into surrounding tissues, and role as a flexible platform for the attachment, proliferation and differentiation of cells. In addition, chondrocytes were shown to maintain their morphology and exhibit enhanced GAG production when seeded on porcine cartilage-derived type II collagen (Nehrer et al. 1997). Alginate and agarose are polysaccharide biomaterials that originate from seaweed and show good biocompatibility and cytocompatibility; however, their poor degradation kinetics and limited potential for functional modification limits their clinical use (Murphy and Sambanis 2001). Fibrin is another natural scaffold that is derived from blood and has been shown to modulate cartilage formation in vivo by facilitating the adhesion of chondrocytes (Fussenegger et al. 2003). Nevertheless, fibrin is mechanically inadequate for cartilage tissue engineering, and its degradation rate is unstable. Thus, it cannot provide a suitable environment as a scaffold for cartilage repair.

Batch-to-batch variations and donor requirements of natural scaffolds have led to the fabrication of synthetic scaffolds with controllable porosity, biodegradability and mechanical functions. Polymeric materials are typically preferred for the creation of synthetic materials due to their ease of fabrication and chemical modification. Poly-glycolic acid (PGA) is a popular material for cartilage autograft studies, and chondrocytes were shown to actively replace the polymer matrix with native ECM components when seeded onto PGA surfaces (Grande et al. 1997). In addition, PGA can form a copolymer with poly-lactic acid (PLA), and this material (polylactic-co-glycolic acid, PLGA) can be engineered to exhibit strong biocompatibility and precise control over degradation rates by adjusting the mixing ratios of PGA and PLA. PLGA offers a flexible environment for cartilage regeneration and was shown to mediate the differentiation of 
adipose-derived adult stem cells into heterogenic cartilage cell populations, which can be utilized for further cartilage engineering applications (Mehlhorn et al. 2009).

\subsubsection{Composite Scaffolds}

Hybrid scaffolds offer novel options for cartilage regeneration by combining the features of individual scaffold elements. For example, chondroitin sulfate (CS) is one of the essential components of cartilage ECM, and the addition of CS to a porous network will enhance the proliferation and differentiation of chondrocytes and provide a degree of bioadhesiveness. Likewise, methyacrylate and aldehyde groups can be integrated into scaffolds in order to establish additional bonds between biomaterials and tissue proteins. Poly ( $\varepsilon$-caprolactone) (PCL) is a common material for such functionalization efforts, as it supports cell attachment, proliferation and ECM production, allowing it to mediate the repair of defect sites and formation of full-thickness cartilage, and the Food and Drug Administration (FDA) approves the clinical use of PCL (Garcia-Giralt et al. 2008). Due to the hydrophobic character of PCL, the cellular recognition and degradation of this polyester is relatively difficult compared to other biomaterials. The integration of polyethylene glycol (as methoxyl poly (ethylene glycol) methyl ether) to PCL enhances the bioactivity of the hydrogel by modulating its hydrophobicity and biodegradability. Graphene oxide (GO) can also be added to these scaffolds to create a more advanced biomaterial with high mechanical strength, conductivity and overall contact area for biological interactions (Lee et al. 2011). The resulting material is a highly porous, elastic, swellable scaffold that has the capacity to convey electricity and is not degraded easily in biological environments, allowing it to establish a suitable long-term environment for cartilage regeneration. (Liao et al. 2015)

Synthetic scaffolds can be further modified through the integration and controlled release of bioactive factors that play important roles in the regeneration process, such as growth factors and hormones. Platelet rich plasma (PRP) contains an elevated level of growth factors, yet its clinical use is limited due to its low mechanical strength and the burst release of its constituent growth factors (Fortier et al. 2011). However, PRP can be mixed with PCL and integrated into a gelatin membrane through the emulsion electrospinning method, producing a composite material that shows high bioactivity and can sustainably release growth factors to stimulate cellular attachment in vitro and support intrinsic cartilage regeneration in vivo (Liu et al. 2015).

In addition to soluble factors, cellular recruitment and integration are also crucial for the success of biomaterial scaffolds. Due to the limited availability of chondrocytes in cartilage tissue, many studies have focused on the use of other cell types to mediate cartilage maintenance and repair following injury. Neonatal chondrocytes (NChons) are the preferred allogeneic cell type for the regeneration of cartilage, but the limited amount of donors prevents their widespread use in clinical settings. Nevertheless, successful culturing environments of NChons were developed using biomaterial scaffolds, such as a 3D hydrogel composed of poly (ethylene glycol diacrylate) and chondroitin sulfate methacrylate. Despite this, however, tissue engineering applications typically rely on MSCs, which are available in abundance, proliferate readily under culture conditions, and can differentiate into chondrocytes. The maintenance of these cells in bioactive scaffolds is an effective means of inducing their differentiation into specific cell lines, and both natural and synthetic scaffolds have been used to support the adhesion, viability, proliferation and differentiation of MSCs. These scaffolds should have a specific set of features to succeed in this role: They must be biocompatible, exhibit minimal immunogenicity and toxicity, possess a microporous nature to ensure oxygen and nutrient transport, allow cells to remodel the scaffold matrix through biodegradation, and alter the stem cell differentiation process to result in a single, specific cell type (Tombuloglu et al. Tombuloglu et al. 2012). Consequently, mimicking the natural ECM of cartilage tissue is a complex process that requires the incorporation of various biomaterial 
components. In addition, MSCs can be derived from multiple sources and exhibit different phenotypes depending on their source. Bone-marrow stem cells (BMSCs) and adipose derived stem cells (ADSCs) are commonly used for cartilage repair, as they are abundant, easy to isolate and naturally inclined towards osteo-chondrogenic differentiation. Co-culture of multiple cell types is also possible, and large neocartilage nodules have been generated by increasing ADSC populations synergistically with NChons, suggesting that the spatial distribution of cells in 3D scaffolds and paracrine signaling between stem cells and chondrocytes are critical parameters for the management of cartilage tissue formation (Lai et al. 2013).

\subsubsection{Peptide-Based Cartilage Engineering Scaffolds}

\subsubsection{Peptide-Edited Hydrogel Systems for Cartilage Regeneration}

More effective scaffold materials can be developed through the incorporation of bioactive epitope-bearing peptide sequences into hydrogels, producing a multifunctional system that contains both the structural architecture and biological signals present in natural scaffolds. Collagen II, for example, is an abundantly found protein in the ECM of cartilage tissue, and the Streptococcal collagen-like II protein was modified with an HA-binding peptide (CGGGRYPISRPRKR) or CS binding peptide (CGGGYKTNFRRYYRF) (Chow et al. 2014) and an MMP7-sensitive crosslinker peptide (CGGGPLELRAGGGC) (Bahney et al. 2011) to create a versatile hydrogel providing a biodegradable and bioactive ECM-mimetic microenvironment. Chondrogenesis was significantly enhanced in human MSCs encapsulated in hydrogels containing the HA-binding peptide, while the CS-binding peptide-containing hydrogel increased MMP7 gene expression and ECM remodeling activity. As such, this multifunctional hydrogel was able to utilize the combined effect of various peptide moieties to stimulate chondrogenic differentiation, and could be used as a minimally invasive method for the repair of cartilage defects (Parmar et al. 2015).

Integrated systems can compensate the deficiencies of their individual components to form mechanically durable and bioactive epitopebearing scaffolds. Demineralized bone matrix is flexible and strong, and presents a threedimensional collagen scaffold. In addition, it is biocompatible, non-immunogenic and, due to its incorporation of various growth factors and cytokines, able to stimulate osteochondrogenic differentiation in vitro ( $\mathrm{Li}$ et al. 2006) and in vivo (Gao et al. 2004). Together with chitosan, demineralized bone forms a mechanically stronger biphasic hydrogel. In addition, chitosan facilitates cell retention and promotes the integration of newly formed cartilage tissue to the defect site due to its sol-gel transition property. This hybrid system can be further modified by an MSC-affine peptide sequence (EPLQLKM), which increases the migration of MSCs to damaged cartilage tissue and allows the biphasic biomaterial to significantly enhance the repair of cartilage defects (Fig. 7.2) (Huang et al. 2014; Meng et al. 2015).

Articular cartilage tissue is composed of superficial, transitional and deep zones. Every zone contains different types of chondrocytes that secrete various amount of proteoglycans and type II collagen (Bhosale and Richardson 2008). Mimicking the layered native structure of articular cartilage may enhance the regeneration of this tissue, and both natural and synthetic biomaterial combinations can be used to form multi-layered functional scaffolds for this purpose. For example, it has been shown that a polyethylene glycol (PEG) based hydrogel, when combined with chondroitin sulfate (CS), matrix metalloproteinase sensitive peptide (MMP-pep) and hyaluronic acid scaffolds, can stimulate the formation of zone-specific chondrocytes from bone-marrow derived stem cells (BMSC) (Bhosale and Richardson 2008). The mechanical and biochemical stimuli provided by distinct scaffold regions led to the formation of specific zones exhibiting characteristic ECM compositions, and three zones of cartilage were created by the multi-layered hydrogel constructs. In particular, the 
A

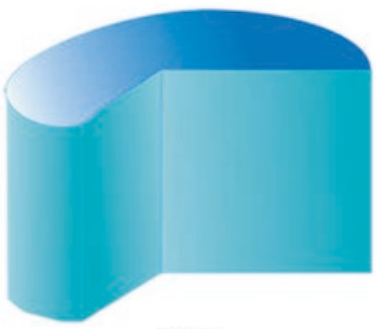

CS

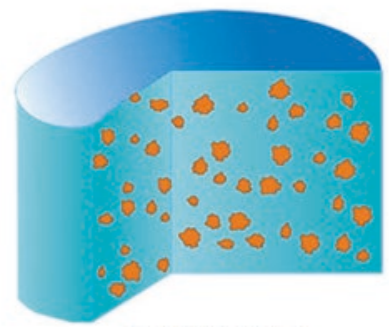

DBM/CS

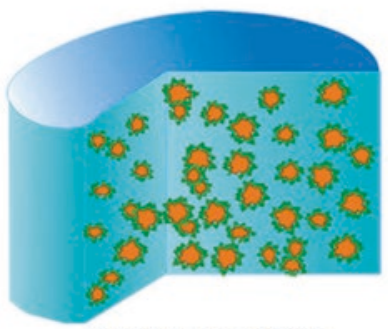

DBM-E7/CS

B

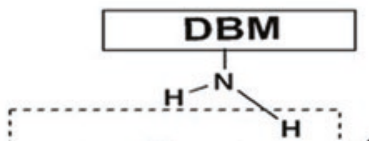

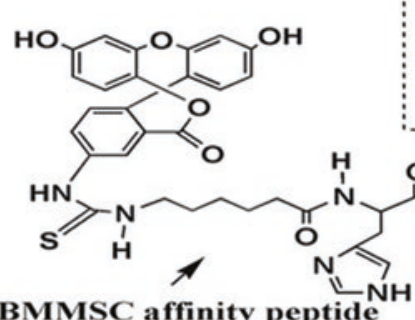

(a)

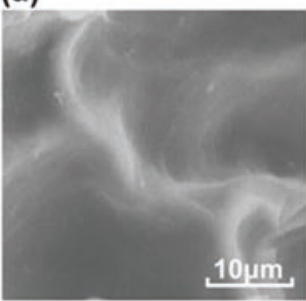

(b)

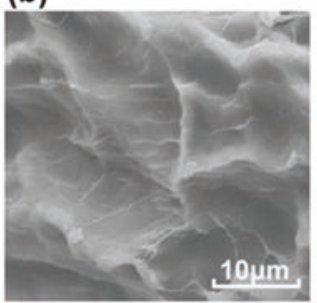

(c)

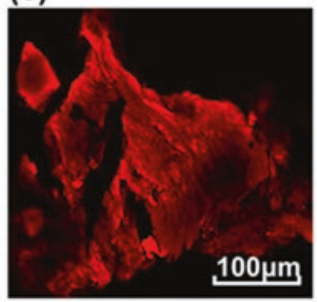

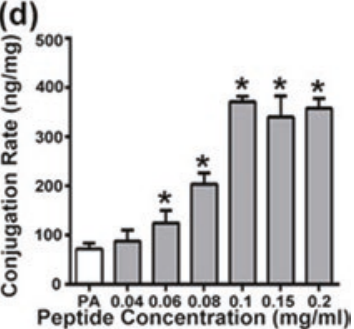

Fig. 7.2 (a) Representation of chitosan scaffold (CS), demineralized bone matrix (DBM) particles and chitosan hydrogel mixture (DBM/CS) scaffold, and combination of mesenchymal stem cells (MSCs) E7 affinity peptide and DBM particles within CS hydrogel scaffold. (b) Chemical structure of bone marrow-derived mesenchymal stem cells (BMMSCs) affinity peptide that is connected to DBM via sulfosuccinimidyl-4-(N-maleimidomethyl) cyclohexane-1-carboxylate (SMCC) cross-linker. (c) Visualization of (a) DBM and (b) DBM-E7 particles via SEM imaging and $(c)$ rhodamine labeled DBM-E7 particles via confocal imaging. (d) Quantification of E7 peptide conjugation on DBM particle scaffold ( $P A$ physical adsorption; *p $<0.05$ vs. PA) (Copyright (C) 2015, Rights Managed by Nature Publishing Group (Meng et al. 2015))
PEG:CS:MMP-pep combination differentiated stem cells into chondrocytes expressing elevated levels of collagen II and low levels of GAG, which is the feature of the superficial zone of cartilage, while the transitional zone was generated with the PEG:CS combination, and the PEG:HA scaffold promoted the formation of the deep zone.
Collagen II expression decreased from the superficial to the deep zone, while the expressions of collagen $\mathrm{X}$ and proteoglycans were enhanced in this direction, which is similar to the situation observed under in vivo conditions and can be applied in clinical settings for the repair of fullthickness cartilage defects (Nguyen et al. 2011). 


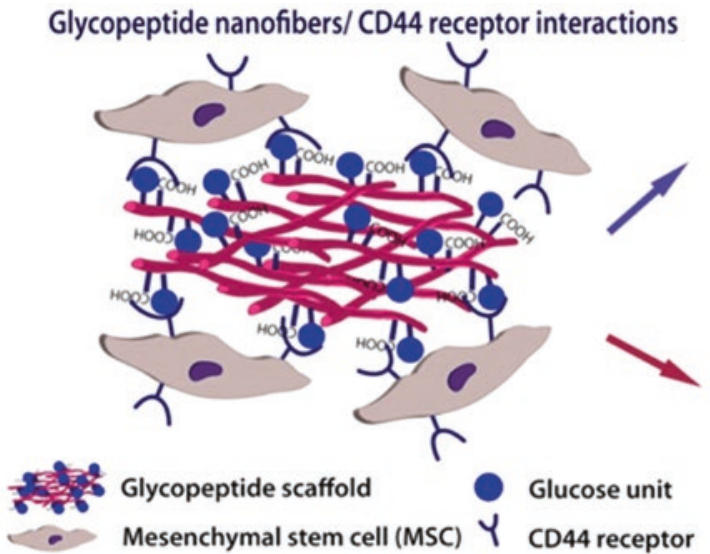

Fig. 7.3 Self- assembled glycopeptide scaffold mimicking hyaluronic acid. Commitment of mesenchymal stem cells to chondrogenesis was visualized by immunolocalization of cartilage specific proteins such as aggrecan, collagen II and Sox 9. Application of glycopeptide scaffold
Cartilage-specific protein expressions by immunolocalization

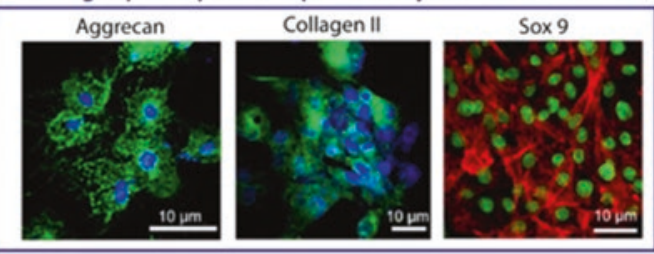

Regenerated tissue after osteochondral defect model creation

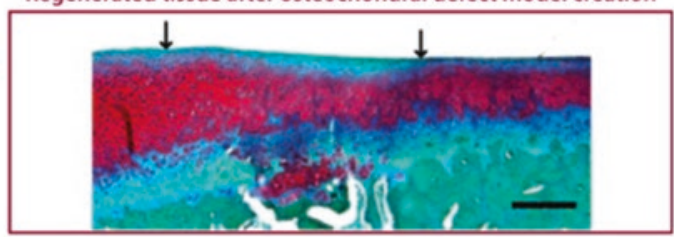

to osteochondral defected cartilage enhanced the regeneration of the tissue (Reprinted with permission from Ustun Yaylaci et al. 2016. Copyright 2016 American Chemical Society)

\subsubsection{Self-Assembled Peptide Gels for Cartilage Regeneration}

Tissue regeneration requires a complex set of biological signals that regulate the recruitment, adhesion, proliferation and differentiation of a broad range of cells. These signals are principally relayed through a fibrous extracellular matrix environment, and supramolecular materials are often designed to mimic this structure through noncovalent self-assembly mechanisms. Noncovalent interactions are preferred for scaffold formation due to their ability to be reversed under relatively mild conditions, which allows both external stimuli and cellular activity to remodel the material during growth and differentiation. In addition, noncovalent interactions are useful for the attachment of bioactive groups that replicate the functions of proteins, growth factors, cytokines and other biomolecules that play important roles during the tissue regeneration process. Peptide-based systems readily selfassemble under physiological conditions into biocompatible, biodegradable and bioactive epitope-bearing networks that mimic the ECM and support the proliferation, adhesion and migration of cells (Boekhoven and Stupp 2014). It was also shown in the study of Yaylaci et al. hyaluronic acid mimetic self-assembled peptide

nanofiber gels enhance the chondrogenesis of MSCs and cartilage regeneration (Fig. 7.3) (Ustun Yaylaci et al. 2016). As such, selfassembled peptide gels have recently emerged as a class of next-generation biomaterials for tissue engineering applications.

Cell-seeded scaffolds for cartilage repair need to provide an appropriate environment for rapid cell division and cartilage-specific ECM deposition. Previously, chondrocytes encapsulated in alginate (Lemare et al. 1998) and agarose (Benya and Shaffer 1982) hydrogels were found to dedifferentiate or fail to preserve their morphologies across extended culturing and passaging periods. However, when encapsulated into self-assembled gels formed by KLD-12 (Ac-KLDLKLDLKLDL-Am) peptide molecules, chondrocytes were able to proliferate extensively while maintaining their phenotype and producing biomechanically functional ECM. The authors suggested that the utility of the system can be further enhanced by encapsulating growth factors within the gel matrix and investigating whether the newly formed cartilage is able to integrate into surrounding tissues (Kisiday et al. 2002). Cell fate can also be directed by controlling the release of soluble factors. Consequently, cellular behaviors can be regulated through the encapsulation of growth fac- 
tors or cytokines into tunable biomaterial networks. Transforming growth factor $\beta 1$ (TGF- $\beta 1$ ) is a critical molecule for the differentiation of mesenchymal stem cells into chondrocytes (Barry et al. 2001), and the Ac-KLDLKLDLKLDL-Am peptide gel system was shown to stimulate the chondrogenic differentiation of bone marrow-derived stromal cells by facilitating the sustained release of this growth factor. Due to their flexible structures, these hydrogels could also be injected easily into nonuniform defect sites, making them promising candidates for the local delivery of cells and growth factors (Kopesky et al. 2014).

The sustained release of TGF- $\beta 1$ was further regulated through the growth factor binding sequence HSNGLPL, which was discovered by phage display method and integrated into the structure of self-assembling TGFBPA hydrogels. These scaffolds were shown to exhibit a gradual release profile for TGF- $\beta 1$, while rapid release was observed from control hydrogels. Human mesenchymal stem cells cultured on TGF- $\beta 1$ encapsulated TGFBPA were viable and committed to the chondrogenic lineage. Further evaluation of the hydrogel was performed on an in vivo microfracture-treated chondral defect model, and TGF- $\beta 1$ binding epitope density was found to strongly affect both the growth factor release profile and the chondrogenic regeneration capacity. In addition, TGFBPA was able to trigger cell differentiation and type II collagen expression even in the absence of encapsulated TGF- $\beta 1$, possibly by retaining endogenously produced TGF- $\beta 1$ (Shah et al. 2010).

Bioactivity of scaffold materials can also be enhanced by the combination of relatively large polymers with small self-assembled peptides. For example, the mixing of hydroxyapatite with the palmitoyl- $\mathrm{V}_{3} \mathrm{~A}_{3} \mathrm{~K}_{3}$-Am peptide sequence was shown to result in the creation of a solid membrane at the interface between the two components, where the negatively-charged HA molecules wereencapsulated by the positively-charged PA networks to form HA-filled sac structures. Viability analyses revealed that gel-filled sacs supported the survival of hMSCs and enhanced collagen type II expression in culture experiments with chondrogenic media supplemented with TGF- $\beta 1$, suggesting that the sacs supply an environment conducive to the chondrogenic differentiation of mesenchymal stem cells (Capito et al. 2008).

Self-assembled peptide nanonetworks are generally more effective inducers of differentiation than other scaffold materials due to their ability to incorporate bioactive sequences in their structure. Chondrogenesis of bone marrow stem cells, for example, was found to be more extensive in self-assembling PA hydrogels compared to bulk hydrogels such as agarose. 3D encapsulation of BMSCs into RAD16-I (AcN- (KLDL) ${ }_{3}-$ $\mathrm{CNH}_{2}$ and RAD16-I ((RADA $\left.)_{4}\right)$ promoted early chondrogenesis as a result of enhanced TGF- $\beta 1$ signaling compared to agarose controls. Since the mechanical characteristics of both systems were similar, morphological differences during chondrogenesis can be attributed to sequence-specific signals. While cell-to-cell contact was present in the RAD16-I systems, multicellular clusters were observed only in the KLD12 hydrogel, and the integration of biochemical and biomechanical signals into the peptide scaffolds was found to allow precise control over the differentiation of MSCs (Kopesky et al. 2010).

\subsection{Conclusion}

Due to the low regenerative capacity of cartilage, even minor tissue defects can cause severe health problems without early intervention. While cartilage repair can be induced through the external administration of stem cells and growth factors, the lack of native blood vessels in cartilage requires the local delivery of the requisite cells and biomolecules, and scaffolds are ideal materials for this purpose. Invasive techniques such as autologous chondrocyte transplantation or microfractures can be used to stimulate cartilage repair, but are associated with severe disadvantages such as fibrosis and collateral tissue damage. Thus, recent advances in cartilage tissue engineering are encouraging from a clinical point of view. The facile synthesis and structural modification of injectable scaffolds is promising for the effective regeneration of large structural 
defects in cartilage. ECM-mimetic, porous and bioactive scaffolds are ideal materials for modulating the signaling networks responsible for cartilage formation and biointegration without eliciting undesirable immune complications. Further achievements in cartilage regeneration are expected to improve the quality of life of the elderly in particular, but it must nevertheless be noted that clinical studies involving peptide-based scaffolds are currently uncommon and that much work needs to be performed to suitably demonstrate the safety and efficiency of this new class of biomaterials for cartilage regeneration.

\section{References}

Ateshian GA (2007) Artificial cartilage: weaving in three dimensions. Nat Mater 6:89-90

Bahney C, Hsu C, Yoo J, West J, Johnstone B (2011) A bioresponsive hydrogel tuned to chondrogenesis of human mesenchymal stem cells. FASEB J 25:1486-1496

Barry F, Boynton R, Liu B, Murphy J (2001) Chondrogenic differentiation of mesenchymal stem cells from bone marrow: differentiation-dependent gene expression of matrix components. Exp Cell Res 268:189-200

Belcher C, Yaqub R, Fawthrop F, Bayliss M, Doherty M (1997) Synovial fluid chondroitin and keratan sulphate epitopes, glycosaminoglycans, and hyaluronan in arthritic and normal knees. Ann Rheum Dis 56:299-307

Benya P, Shaffer J (1982) Dedifferentiated chondrocytes reexpress the differentiated collagen phenotype when cultured in agarose gels. Cell 30:215-224

Bhosale A, Richardson J (2008) Articular cartilage: structure, injuries and review of management. Br Med Bull 87:77-95

Boekhoven J, Stupp S (2014) 25th anniversary article: supramolecular materials for regenerative medicine. Adv Mater 26:1642-1659

Brandt KD, Smith GN, Simon LS (2000) Intraarticular injection of hyaluronan as treatment for knee osteoarthritis: what is the evidence? Arthritis Rheum 43:1192-1203

Capito R, Azevedo H, Velichko Y, Mata A, Stupp S (2008) Self-assembly of large and small molecules into hierarchically ordered sacs and membranes. Science 319:1812-1816

Chow L, Armgarth A, St-Pierre J, Bertazzo S, Gentilini C, Aurisicchio C, McCullen S, Steele J, Stevens M (2014) Peptide-directed spatial organization of biomolecules in dynamic gradient scaffolds. Adv Healthc Mater 3:1381-1386
Eyre D (2002) Collagen of articular cartilage. Arthritis Res 4:30-35

Felson DT, Zhang Y, Hannan MT, Naimark A, Weissman B, Aliabadi P, Levy D (1997) Risk factors for incident radiographic knee osteoarthritis in the elderly: the Framingham study. Arthritis Rheum 40:728-733

Fortier L, Barker J, Strauss E, McCarrel T, Cole B (2011) The role of growth factors in cartilage repair. Clin Orthop Relat Res 469:2706-2715

Fussenegger M, Meinhart J, Hobling W, Kullich W, Funk S, Bernatzky G (2003) Stabilized autologous fibrinchondrocyte constructs for cartilage repair in vivo. Ann Plast Surg 51:493-498

Gaissmaier C, Koh JL, Weise K (2008) Growth and differentiation factors for cartilage healing and repair. Injury 39:88-96

Gao J, Knaack D, Goldberg V, Caplan A (2004) Osteochondral defect repair by demineralized cortical bone matrix. Clin Orthop Relat Res 427:S62-S66

Garcia-Giralt N, Izquierdo R, Nogues X, Perez-Olmedilla M, Benito P, Gomez-Ribelles J, Checa M, Suay J, Caceres E, Monllau J (2008) A porous PCL scaffold promotes the human chondrocytes redifferentiation and hyaline-specific extracellular matrix protein synthesis. J Biomed Mater Res A 85:1082-1089

Ge Z, Li C, Heng B, Cao G, Yang Z (2012) Functional biomaterials for cartilage regeneration. J Biomed Mater Res A 100:2526-2536

Gillogly SD, Voight M, Blackburn T (1998) Treatment of articular cartilage defects of the knee with autologous chondrocyte implantation. J Orthop Sports Phys Ther 28:241-251

Grande D, Halberstadt C, Naughton G, Schwartz R, Manji R (1997) Evaluation of matrix scaffolds for tissue engineering of articular cartilage grafts. J Biomed Mater Res 34:211-220

Grigolo B, Roseti L, Fiorini M, Fini M, Giavaresi G, Aldini N, Giardino R, Facchini A (2001) Transplantation of chondrocytes seeded on a hyaluronan derivative (Hyaff@-11) into cartilage defects in rabbits. Biomaterials 22:2417-2424

Grotle M, Hagen KB, Natvig B, Dahl FA, Kvien TK (2008) Obesity and osteoarthritis in knee, hip and/or hand: an epidemiological study in the general population with 10 years follow-up. BMC Musculoskelet Disord 9:132

Han L, Grodzinsky A, Ortiz C (2011) Nanomechanics of the cartilage extracellular matrix. Ann Rev Mater Res 41:133-168

Heinegård D, Saxne T (2011) The role of the cartilage matrix in osteoarthritis. Nat Rev Rheumatol 7:50-56

Huang H, Zhang X, Hu X, Shao Z, Zhu J, Dai L, Man Z, Yuan L, Chen H, Zhou C, Ao Y (2014) A functional biphasic biomaterial homing mesenchymal stem cells for in vivo cartilage regeneration. Biomaterials 35:9608-9619

Kisiday J, Jin M, Kurz B, Hung H, Semino C, Zhang S, Grodzinsky A (2002) Self-assembling peptide hydrogel fosters chondrocyte extracellular matrix produc- 
tion and cell division: implications for cartilage tissue repair. Proc Nat Acad Sci USA 99:9996-10001

Kock NB, Smolders JM, Van Susante JL, Buma P, Van Kampen A, Verdonschot N (2008) A cadaveric analysis of contact stress restoration after osteochondral transplantation of a cylindrical cartilage defect. Knee Surg Sports Traumatol Arthrosc 16:461-468

Kopesky P, Vanderploeg E, Sandy J, Kurz B, Grodzinsky A (2010) Self-assembling peptide hydrogels modulate in vitro chondrogenesis of bovine bone marrow stromal cells. Tissue Eng Part A 16:465-477

Kopesky PW, Byun S, Vanderploeg EJ, Kisiday JD, Frisbie DD, Grodzinsky AJ (2014) Sustained delivery of bioactive TGF- $\beta 1$ from self-assembling peptide hydrogels induces chondrogenesis of encapsulated bone marrow stromal cells. J Biomed Mater Res A 102:1275-1285

Kreuz PC, Steinwachs MR, Erggelet C, Krause SJ, Konrad G, Uhl M, Südkamp N (2006) Results after microfracture of full-thickness chondral defects in different compartments in the knee. Osteoarthrithis Cartilage 14:1119-1125

Lai J, Kajiyama G, Smith R, Maloney W, Yang F (2013) Stem cells catalyze cartilage formation by neonatal articular chondrocytes in 3D biomimetic hydrogels. Sci Rep 3:3553

Lee W, Lim C, Shi H, Tang L, Wang Y, Lim C, Loh K (2011) Origin of enhanced stem cell growth and differentiation on graphene and graphene oxide. ACS Nano 5:7334-7341

Lemare F, Steimberg N, Le Griel C, Demignot S, Adolphe M (1998) Dedifferentiated chondrocytes cultured in alginate beads: restoration of the differentiated phenotype and of the metabolic responses to interleukin-1 $\beta$. J Cell Physiol 176:303-313

Li X, Jin L, Balian G, Laurencin C, Anderson D (2006) Demineralized bone matrix gelatin as scaffold for osteochondral tissue engineering. Biomaterials 27:2426-2433

Li WJ, Chiang H, Kuo TF, Lee HS, Jiang CC, Tuan RS (2009) Evaluation of articular cartilage repair using biodegradable nanofibrous scaffolds in a swine model: a pilot study. J Tissue Eng Regen Med 3:1-10

Liao J, Qu Y, Chu B, Zhang X, Qian Z (2015) Biodegradable CSMA/PECA/graphene porous hybrid scaffold for cartilage tissue engineering. Sci Rep 5:9879

Lin Y, Luo E, Chen X, Liu L, Qiao J, Yan Z, Li Z, Tang W, Zheng X, Tian W (2005) Molecular and cellular characterization during chondrogenic differentiation of adipose tissue-derived stromal cells in vitro and cartilage formation in vivo. J Cell Mol Med 9:929-939

Liu J, Nie H, Xu Z, Guo F, Guo S, Yin J, Wang Y, Zhang C (2015) Construction of PRP-containing nanofibrous scaffolds for controlled release and their application to cartilage regeneration. J Mater Chem B 3:581-591
Mehlhorn A, Zwingmann J, Finkenzeller G, Niemeyer P, Dauner M, Stark B, Sudkamp N, Schmal H (2009) Chondrogenesis of adipose-derived adult stem cells in a poly-lactide-co-glycolide scaffold. Tissue Eng Part A 15:1159-1167

Meng Q, Man Z, Dai L, Huang H, Zhang X, Hu X, Shao Z, Zhu J, Zhang J, Fu X, Duan X, Ao Y (2015) A composite scaffold of MSC affinity peptide-modified demineralized bone matrix particles and chitosan hydrogel for cartilage regeneration. Sci Rep 5:17802

Moreland LW (2003) Intra-articular hyaluronan (hyaluronic acid) and hylans for the treatment of osteoarthritis: mechanisms of action. Arthritis Res Ther 5:54-67

Murphy C, Sambanis A (2001) Effect of oxygen tension and alginate encapsulation on restoration of the differentiated phenotype of passaged chondrocytes. Tissue Eng 7:791-803

Nehrer S, Breinan H, Ramappa A, Shortkroff S, Young G, Minas T, Sledge C, Yannas I, Spector M (1997) Canine chondrocytes seeded in type I and type II collagen implants investigated in vitro. J Biomed Mater Res 38:95-104

Nguyen L, Kudva A, Saxena N, Roy K (2011) Engineering articular cartilage with spatially-varying matrix composition and mechanical properties from a single stem cell population using a multi-layered hydrogel. Biomaterials 32:6946-6952

Parmar P, Chow L, St-Pierre J, Horejs C, Peng Y, Werkmeister J, Ramshaw J, Stevens M (2015) Collagen-mimetic peptide-modifiable hydrogels for articular cartilage regeneration. Biomaterials 54:213-225

Pearle AD, Warren RF, Rodeo SA (2005) Basic science of articular cartilage and osteoarthritis. Clin Sports Med 24:1-12

Prakash D, Learmonth D (2002) Natural progression of osteo-chondral defect in the femoral condyle. Knee 9:7-10

Shah RN, Shah NA, Del Rosario Lim MM, Hsieh C, Nuber G, Stupp SI (2010) Supramolecular design of self-assembling nanofibers for cartilage regeneration. Proc Natl Acad Sci U S A 107:3293-3298

Slomianka L (2009) Blue histology - skeletal tissues - cartilage. www.lab.anhb.uwa.edu.au/mb140/ CorePages/Cartilage/Cartil.htm

Taipaleenmaki H (2010) Factors regulating chondrogenic differentiation. University of Turku, Finland

Tombuloglu A, Tekinay AB, Guler MO (2012) Materials for articular cartilage regeneration. Recent Pat Biomed Eng 5:187-199

Ustun Yaylaci S, Sardan Ekiz M, Arslan E, Can N, Kilic E, Ozkan H, Orujalipoor I, Ide S, Tekinay AB, Guler MO (2016) Supramolecular GAG-like self-assembled glycopeptide nanofibers induce chondrogenesis and cartilage regeneration. Biomacromolecules 17:679-689 